\title{
Biology of corneal fibrosis: soluble mediators, integrins, and extracellular vesicles
}

\author{
Tina B. McKay ${ }^{1}$ - Audrey E. K. Hutcheon ${ }^{1}$. James D. Zieske ${ }^{1}$
}

Received: 26 September 2019 / Revised: 1 November 2019 / Accepted: 27 November 2019 / Published online: 12 December 2019

(c) The Royal College of Ophthalmologists 2019

\begin{abstract}
Corneal fibrosis develops in response to injury, infection, postsurgical complications, or underlying systemic disease that disrupts the homeostasis of the tissue leading to irregular extracellular matrix deposition within the stroma. The mechanisms that regulate corneal scarring are focused heavily on the canonical transforming growth factor- $\beta$ pathway and relevant activators, and their role in promoting myofibroblast differentiation. In this paper, we discuss the biochemical pathways involved in corneal fibrosis in the context of different injury models-epithelial debridement, superficial keratectomy, and penetrating incision. We elaborate on the interplay of the major pro-fibrotic factors involved in corneal scar development (e.g., transforming growth factor- $\beta 1$, thrombospondin-1, and $\alpha \nu \beta 6$ ), and explore a novel role for extracellular vesicles secreted by the wounded epithelium and the importance of the basement membrane.
\end{abstract}

\section{Introduction}

Corneal scarring may be caused by infection, injury, or disease and result in restricted passage of light that reaches the retina, thus often leading to detrimental effects on visual acuity. The World Health Organization estimates that corneal opacity accounts for over $4 \%$ of blindness worldwide leading to over 1.5-2 million cases each year [1,2]. Todate, the only effective treatment for vision loss due to corneal scarring is corneal transplantation.

In general terms, corneal haze that reduces the transparency of the tissue may be caused by sub-clinical oedema, inflammatory cell flux, or the presence of fibroblasts or myofibroblasts that deposit modest amounts of extracellular matrix (ECM). The severity of scar formation can be evaluated by assessing the corneal backscatter as a measure of the mean reflectivity of the tissue. Recovery of corneal transparency following the presentation of corneal haze may occur with the remodelling and removal of excess ECM over the course of months to years. This regenerative

Tina B. McKay

Tina_McKay@meei.harvard.edu

1 Schepens Eye Research Institute/Massachusetts Eye and Ear, Department of Ophthalmology, Harvard Medical School, Boston, MA 02114, USA process is determined by the severity of the original injury and the recovery of proper barrier function, which influence the inflammatory cell influx and penetrance of pro-fibrotic factors that promote the persistence of myofibroblasts. Severe scar tissue, as a result of excess deposition of a highly disorganized ECM, gives rise to irregular fibril structure and organization that cannot readily be remodelled under physiological conditions. Therefore, close regulation of the pathways involved in tissue regeneration and wound closure is required to maintain corneal transparency, as well as prevent infection and permanent tissue damage.

\section{Corneal injury models}

The mechanisms important in corneal wounding have been largely unravelled by studies using three major corneal injury models: (1) Epithelial debridement; (2) Superficial keratectomy; (3) Penetrating incision (Fig. 1) [3, 4]. The wound size varies between species depending on corneal diameter and thickness in the model organism. In this review, we focus on wound models using mice, given the broad availability of knockout models to study the role of specific factors involved in corneal wound healing. Epithelial debridement generally involves the removal of the epithelium from the central cornea via manual scraping with a blunt blade, while leaving the basement membrane intact. In mice, this type of wound ranges from 1 to $2 \mathrm{~mm}$ in 
Fig. 1 Mechanisms involved in corneal fibrosis following injury. a Epithelial debridement leaves an intact basement membrane, which may play a role in restricting diffusion of pro-fibrotic factors, including TGF- $\beta 1$ and EVs secreted by the wounded epithelium. b A more severe wound induced by a keratectomy, which removes the epithelium, basement

membrane, and a portion of the anterior stroma, often leads to corneal fibrosis characterized by myofibroblast differentiation. c A penetrating incision, which damages both the anterior and posterior corneal surfaces, leads to severe scar formation likely as a result of aqueous humor flux into the cornea.

a

\section{Epithelial debridement}

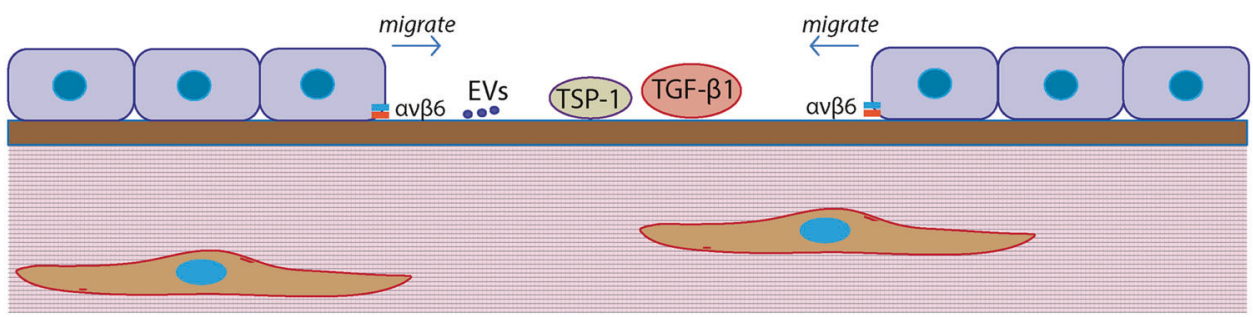

b

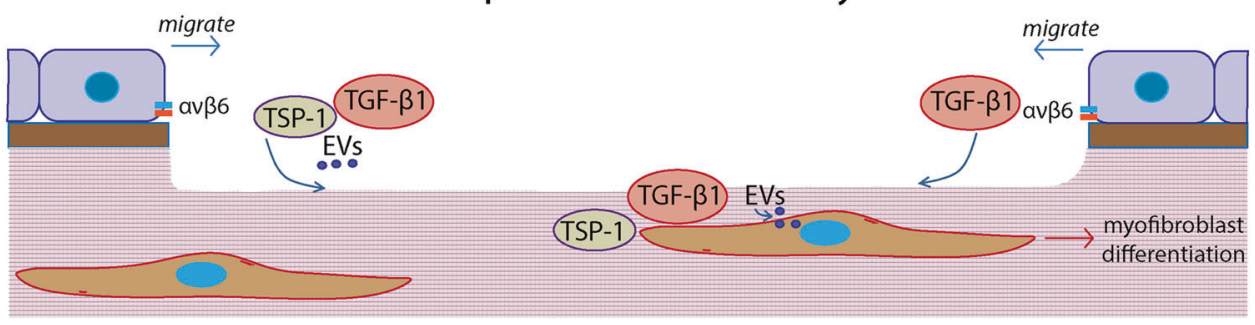

C

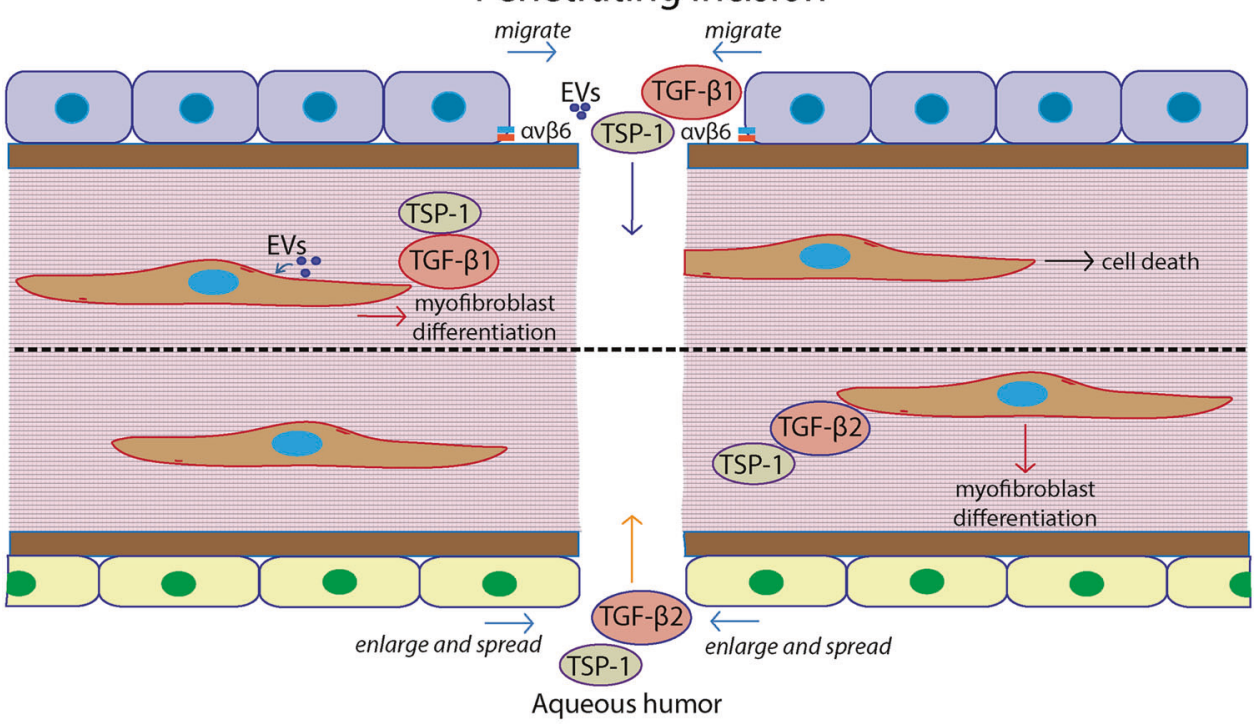

Key: $\square$ corneal epithelial cell

$\int$ corneal keratocyte

- corneal endothelial cell

basement membrane $\equiv$ stromal ECM

diameter and 10-20 $\mu \mathrm{m}$ deep [4]. Generally, the corneal epithelium recovers from a debridement within 18-48 h, depending on the species, with no stromal or inflammatory cell involvement or scar development, though recurrent erosion of the epithelium may occur in mice [5]. In contrast, a superficial keratectomy is performed by placing a trephine on the central cornea, applying gentle pressure, and rotating 2-3 times to demarcate the region of injury, followed by the manual removal of the epithelium, basement membrane, and anterior stroma with sharp forceps [3]. A keratectomy tends to be $1.5-2 \mathrm{~mm}$ in diameter in mice and ranges between 20 and $50 \mu \mathrm{m}$ in depth, depending on species [4]. Following a superficial keratectomy, the basement membrane generally regenerates by 1 month in mice [6] and rabbits [7]. This type of wound will form a scar; however, the abundance of myofibroblasts usually peak at 1 month in rabbits and resolve by 3 months [8]. The most severe corneal injury model of these three is a penetrating incision, which in mice is induced using a 1-mm length knife that is applied to the central cornea with gentle pressure so that the knife penetrates through the entire cornea (epithelium, basement membrane, stroma, Descemet's membrane, and 
endothelium) through to the aqueous humor [3]. This type of injury forms a gap that takes about 40 days to heal in mice. A large number of myofibroblasts are associated with this type of wound and are observed within the region of injury [9], thus leading to significant scar development that does not resolve with time [10].

The potentiality for corneal scar development is largely dependent on the integrity of the epithelial and endothelial layers and their respective basement membranes, which influence the penetrance of pro-fibrotic factors [e.g., transforming growth factor- $\beta$ (TGF- $\beta$ ), thrombospondin-1 (TSP1), and extracellular vesicles (EVs)] into the stroma (Fig. 1). Injuries, such as epithelial debridement, which leave the basement membrane intact, usually do not result in scar formation; however, more severe injuries where the basement membrane(s) may be damaged (e.g., superficial keratectomy or penetrating incision), often result in a corneal scar, which may not resolve depending upon the severity of the wound. Scarring susceptibility may also be influenced by species-differences in corneal structure, such as the presence or absence of Bowman's layer in humans and mice, respectively. Bowman's layer in humans may provide some protection against corneal scarring simply by increasing the spatial distance between keratocytes found within the stroma and the ocular surface, thus limiting direct cell damage to the stroma caused by superficial ocular trauma. However, the basement membrane found in both humans and mice likely plays a more significant role in preventing scar development via direct protein-protein interactions between basement membrane proteins and secreted pro-fibrotic factors that are conserved between species. Certain pro-fibrotic factors, such as TSP-1, contain binding domains to basement membrane proteins (e.g., heparan sulfate proteoglycans) that may restrict diffusion through the basal lamina, thus hampering growth factormediated keratocyte activation. These potential binding interactions between pro-fibrotic factors and the basement membrane emphasize the importance of this conserved cellfree layer in scar development.

In this review, we discuss myofibroblast differentiation, TGF- $\beta 1$, TSP- 1 , and integrin $\alpha \nu \beta 6$, and their roles in corneal wound repair and regeneration in the mouse model, thereby examining the role of the corneal basement membrane and injury severity in scar development independent of the Bowman's layer.

\section{Myofibroblast differentiation}

Myofibroblasts are a contractile cell type that are predominately derived in the cornea from resident keratocytes which have undergone activation in response to pro-fibrotic factors secreted following injury, such as TGF- $\beta 1$, platelet- derived growth factor, and connective tissue growth factor, thus promoting myofibroblast differentiation. The term "myofibroblast differentiation" is utilized in this sense according to the definition proposed by Tomasek et al., in reference to "the elaboration of particular characteristics that are expressed by an end-stage cell type or by a cell en route to becoming an end-stage cell" [11]; in this case, differentiation refers to the process leading to the expression of key myofibroblast markers, such as $\alpha$-smooth muscle actin $(\alpha-$ SMA $)$ and collagen type III, rather than cell commitment. Following a full-thickness penetrating incision of the cornea, $\alpha$-SMA-positive cells appear within the corneal stroma at 7 days post injury and disappear by 28-42 days [9]. The presence of myofibroblasts during corneal wound healing provides an essential role in wound closure via cellular and tissue contraction, as well as acute ECM deposition, to limit intraocular exposure to the external environment. In addition, myofibroblasts are temporally and spatially regulated by key pro-fibrotic factors, which influence the rate and degree of corneal repair following injury.

The downside of myofibroblasts is they are the primary cell responsible for irregular ECM deposition and scar development in the cornea. As keratoctyes differentiate to myofibroblasts, a loss in cellular transparency occurs due to the downregulation of crystallin proteins, aldehyde dehydrogenase class 1 , and transketolase, which are highly expressed in the resident keratocyte [12]. The loss in tissue transparency is further accompanied by excess ECM deposition by myofibroblasts. The development of a corneal scar or opacity within the visual axis is detrimental to vision, and the goal of many scientists is to limit or reverse fibrosis. One idea to assist in reducing scar development is to focus on TGF- $\beta 1$, which is a potent inhibitor of interleukin-1-induced apoptosis of myofibroblasts; therefore, if TGF- $\beta 1$ is restored to its basal levels within the stroma, the myofibroblasts within the healing corneal stroma would then be allowed to undergo apoptosis, thus potentially limiting the opacity caused by wounding [13]. Another possible avenue of treatment would be to stimulate the mature myofibroblasts to revert back to a quiescent phenotype [14]; however, to the authors' knowledge, there are no reports to-date of transdifferentiation of myofibroblasts to keratocytes within the corneal stroma in vivo.

Studies also have reported that myofibroblasts found within the cornea postkeratectomy may also originate from fibrocytes [15], which are bone-marrow-derived cells found in circulation that express markers of hematopoietic cells (e.g., CD34 ${ }^{+}, \mathrm{CD} 9^{+}$, and $\mathrm{CD} 90 / \mathrm{Th}^{+}$), as well as the leukocyte marker, $\mathrm{CD} 45^{+}$. Roughly, $30-50 \%$ of resident myofibroblasts in mice appear to be derived from fibrocytes, which maintain co-expression of CD45, vimentin, and $\alpha$ SMA up to 21 days post-wounding, at which point they likely undergo apoptosis [15]. 


\section{Pro-fibrotic factors involved in corneal scarring}

\section{TGF- $\beta$}

TGF- $\beta$ is a growth factor that regulates diverse pathways in the body, including cellular differentiation, immune modulation, and ECM deposition and remodeling. It is secreted in an inactive form that is bound to the latency-associated protein (LAP), and a number of ligands-TSP-1, integrins, plasmin, and matrix metalloproteinases- 2 and -9 - bind to the LAP, which leads to the release of active TGF- $\beta$ [16].

There are three known isoforms of TGF- $\beta$ expressed in mammalian cells (TGF- $\beta 1,-\beta 2$, and $-\beta 3$ ) and each isoform performs diverse functions depending on the cell type and localization. For example, the presence of TGF- $\beta 1$ is required for proper growth and development. TGF- $\beta 1^{-1-}$ mice exhibit high intrauterine lethality $(>50 \%)$, and those that are born, usually do not survive past 3-5 weeks postnatal due to chronic inflammation and necrosis [17]. In contrast, overexpression of TGF- $\beta 1$ by linkage to an epidermal-specific promoter in mice leads to death $<24 \mathrm{~h}$ postnatal due to severe epithelial defects and heart abnormalities [18]. Furthermore, overexpression of TGF- $\beta 1$ within the central nervous system leads to hydrocephalus and increased ECM production, including elevated laminin and fibronectin expression [19]. Clearly, close regulation of TGF- $\beta 1$ levels is required for proper organismal growth and development.

In addition to being vitally important during embryonic development, TGF- $\beta$ also plays an essential role in mediating the response to injury in adult organisms. As seen with corneal wound healing, TGF- $\beta 1$ levels dramatically increase within the tear film [20], with parallel elevation in the primary TGF- $\beta 1$ receptors, TGF- $\beta$ RI and $-\beta$ RII [21]. Free-diffusion of TGF- $\beta 1$ and $-\beta 2$ to the stroma in injuries involving the disruption of basement membrane integrity (Fig. 1b), such as photorefractive keratectomy, has been purported as a mechanism of fibrosis by driving myofibroblast differentiation and persistence [16]. In agreement, stimulation of keratocytes and fibroblasts with subnanomolar concentrations of TGF- $\beta 1$ in vitro leads to myofibroblast differentiation [22]. This mechanism of fibrosis is primarily through the canonical signalling pathway, where TGF- $\beta 1$ or $-\beta 2$ bind to the serine-threonine kinase receptors, TGF- $\beta$ RI and $-\beta$ RII, which leads to the recruitment of the cytosolic proteins, SMAD2 and 3, to the plasma membrane. SMAD2 and 3 are then phosphorylated, bind to SMAD4, translocate to the nucleus, and activate the transcription of genes associated with collagen deposition and stress fiber formation [23]. The less abundant isoform, TGF- $\beta 3$, has been identified to play an important role during development [24] and scarless tissue regeneration [25], which is likely mediated via a noncanonical pathway.
Studies for blocking fibrosis or slowing its progression are currently being investigated at the TGF- $\beta$ level, as well as further down the signalling pathway. Inhibition of TGF- $\beta$ signalling via overexpression of the regulatory SMAD7 following corneal injury has been found to be associated with blunted scar formation [26]. In addition, blocking the downstream effectors of the canonical TGF- $\beta$ pathway, including TSP- 1 and $\alpha \nu \beta 6$, have likewise been found to be associated with the inhibition of myofibroblast differentiation, though a parallel delay in wound closure has been observed, supporting a fundamental role for this pathway during corneal regeneration $[6,27,28]$.

\section{TSP-1}

TSP-1 is a large homotrimeric, multidomain protein with a number of binding partners, including heparan sulfate proteoglycans, collagen, fibronectin, pro-TGF- $\beta 1$, and integrins [29]. TSP-1 plays an important role in ECM deposition and myofibroblast differentiation via regulation of TGF- $\beta$ activity [30]. Binding of TSP-1 to the LAP-TGF- $\beta$ complex results in the release of active TGF- $\beta$ [31], which then binds to its respective receptors (Fig. 2a). Basal expression of TSP-1 in the cornea is primarily localized to the endothelium, though injury via debridement or superficial keratectomy leads to induced expression at the basal epithelium and anterior stroma, respectively [32]. The upregulation in TSP-1 postwounding may influence the stromal response depending on the presence or absence of the basement membrane, as TSP-1 contains binding domains for heparan sulfate proteoglycans (e.g., perlecan and agrin) and collagen (e.g., type IV), which, in addition to laminin and nidogen, are major components of the epithelial basement membrane [33].

In agreement with the role of TSP-1 in promoting myofibroblast differentiation in the absence of the basement membrane, co-localization of TSP- 1 and $\alpha$-SMA in the wounded region following a keratectomy or penetrating incision is readily apparent in the anterior stroma [27, 32]. Furthermore, TSP- $1^{-1-}$ mice show reduced $\alpha$-SMA expression by 4 days post keratectomy [32] and lower numbers of myofibroblasts following penetrating incision [27] compared to wild-type mice, in a process that is likely a result of blunted TGF- $\beta$-mediated activation due to the absence of TSP-1. Consistent with the importance of myofibroblasts in tissue contraction and subsequent closure, TSP- ${ }^{-1-}$ mice also exhibit poor corneal repair following a penetrating incision, characterized by the presence of a gaping wound and persistent oedema [27]. The functional role of TSP-1 in the uninjured adult cornea is unknown but may influence corneal endothelial cell migration [34]. Moreover, deficiency in TSP-1 expression leads to defective endothelial barrier regeneration following injury, and Descemet's membrane does not appear to re-form in TSP- $1^{-1-}$ 

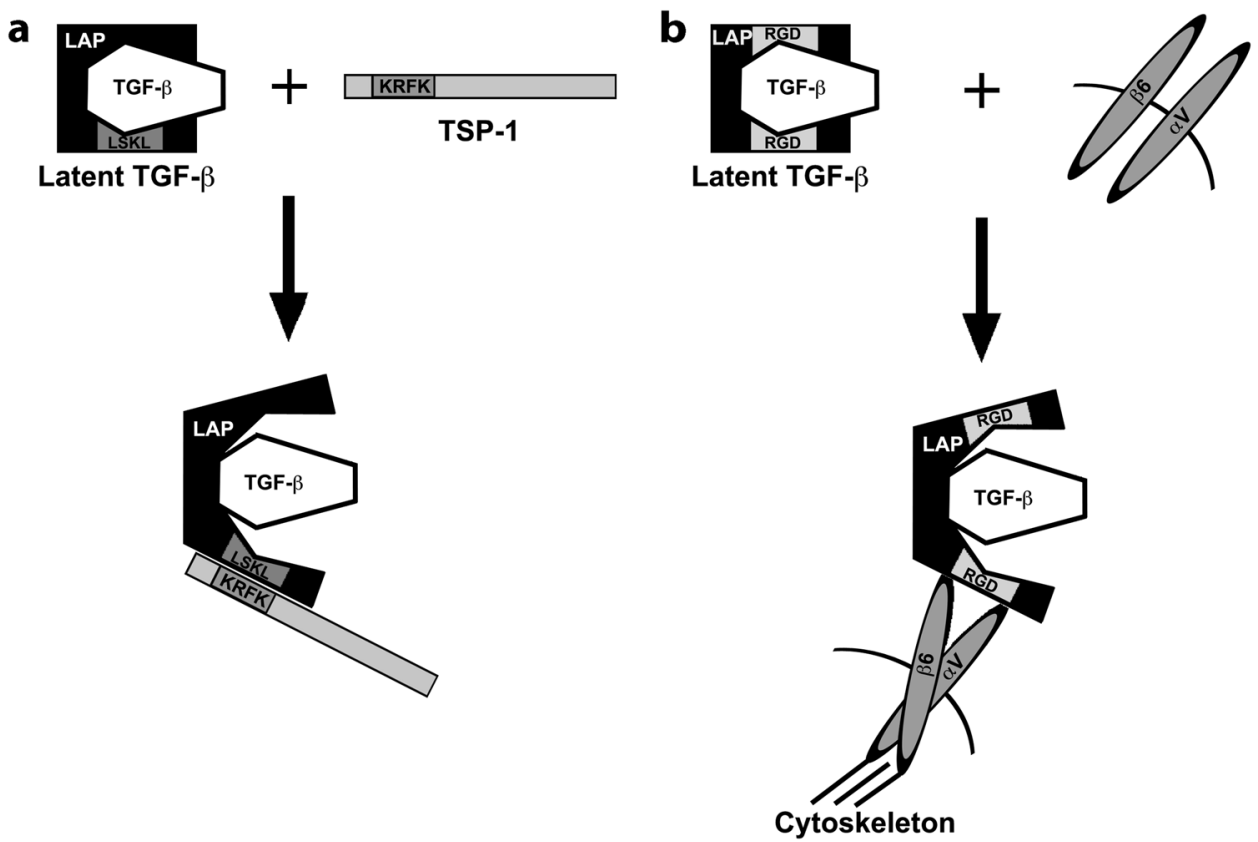

Fig. 2 Activation of TGF- $\beta$ by TSP-1 and $\alpha \nu \boldsymbol{\beta} 6$. TGF- $\beta 1$ is secreted as an inactive protein bound to LAP. a TSP-1 binds to the LAP protein via interactions between the Lys-Arg-Phe-Lys (KRFK)-peptide sequence found within the Type 1 repeats of TSP-1 and Leu-Ser-LysLeu (LSKL) domain of LAP. b The integrin, $\alpha \nu \beta 6$, may bind to the Arg-Gly-Asp (RGD)-domain found in LAP leading to release of active

mice, as evident by the development of severe edema compared to wild-type mice, which recover barrier integrity by 2 weeks post penetrating injury [27]. These studies suggest that TSP-1 is a dominant factor in promoting scar development and corneal repair post wounding.

$\operatorname{av} \beta 6$

A number of integrins are expressed by the corneal epithelium, including $\alpha 2 \beta 1, \alpha 3 \beta 1, \alpha 6 \beta 1$, and $\alpha \nu \beta 5$ [35]. Similar to TSP-1, $\alpha \nu \beta 6$ is expressed by the epithelium primarily after corneal injury, where it plays an important role in mediating epithelial-cell migration and adhesion via binding to the Arg-Gly-Asp (RGD) domain of fibronectin [36]. The role of $\alpha \nu \beta 6$ in wound healing is also associated with its regulatory function of TGF- $\beta$ signalling via the release of active TGF- $\beta$ by binding to the RGD domain of LAP (Fig. 2b) [37]. In addition, mice with $\alpha \nu \beta 6$ deficiency $\left(\beta 6^{-I-}\right)$ have a significant delay in corneal wound healing following a keratectomy, with reduced epithelial basement membrane formation and adhesion, which is likely attributed to lower laminin production [6]. Moreover, morphological defects in corneal structure in the form of blister-like structures on the surface, as a result of poor epithelial adhesion to the underlying basement membrane, can be found in the $\beta 6^{-1-}$ mice by 1 week post keratectomy [6]. Given the prominent role of TGF- $\beta$ in tissue contraction and

TGF- $\beta 1$, which is then able to bind to TGF- $\beta$ RI and $-\beta$ RII leading to phosphorylation of the SMAD proteins. Translocation of this complex leads to the transcription of genes associated with myofibroblast differentiation, including $\alpha$-SMA and collagen type III [23, 43]. Adapted from $[31,44]$ and re printed with permission.

scar development, $\beta 6^{-/-}$mice also exhibit delayed wound closure, which may contribute to increased risk for infection, as well as scarring accompanied by inflammatory cell influx.

While the presence or absence of $\alpha \nu \beta 6$ clearly influences regeneration of the corneal epithelium, this integrin also directly influences myofibroblast differentiation following injury. The spatial and temporal expression of $\alpha \nu \beta 6$ by $24 \mathrm{~h}$ post wounding preludes the expression of $\alpha$-SMA and TSP-1. Following induction of a more severe wound induced via a penetrating incision, the absence of $\alpha \nu \beta 6$ likewise leads to a subsequent delay in both $\alpha$-SMA and TSP-1 peak expression by 28 days compared to 14 days in wild-type mice, supporting a functional role for $\alpha \nu \beta 6$ expression as a pro-fibrotic factor [28]. These results suggest that $\alpha \nu \beta 6$ may initiate the repair response that leads to wound closure with direct regulation of downstream mediators, TSP-1 and TGF- $\beta 1$.

\section{Future perspectives: role of EVs}

EVs are secreted membrane-bound vesicles composed of different sub-types, including microvesicles, ectosomes, exosomes, and others, that may serve important roles in cellular communication [38]. The contents contained within EVs are enriched in select peptides, proteins, and microRNA that may differentially regulate the transcriptional and translational properties of the target cell. A recent study has suggested that EVs isolated from limbal stromal cells promote limbal 

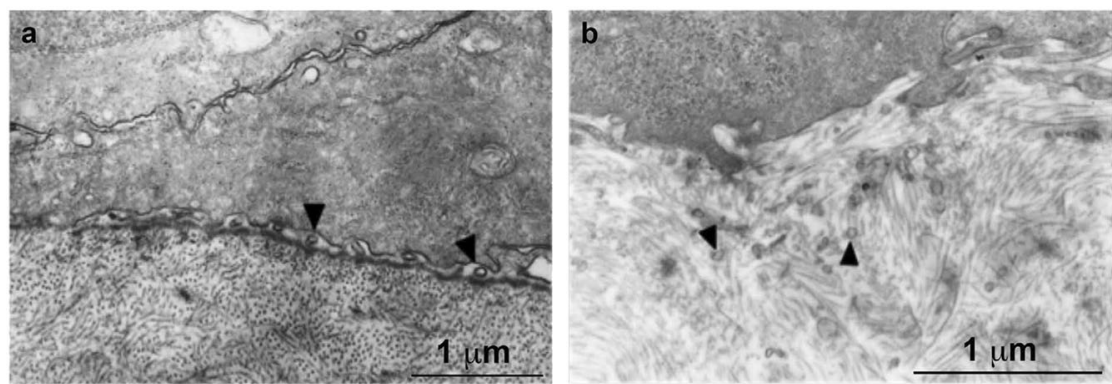

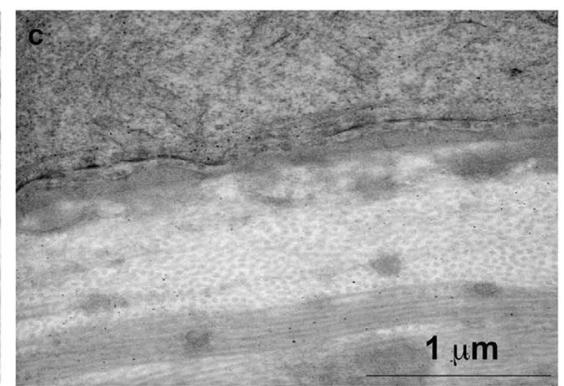

an uninjured rat cornea. Arrowheads denote the appearance of secreted EVs. Figure obtained from [41] based on a Creative Commons Attribution 4.0 International License.

- Activation occurs via release from LAP may be mediated by a number of factors, including TSP-1 and $\alpha \nu \beta 6$.

- Promotes myofibroblast differentiation via the canonical TGF- $\beta 1$ signalling pathway involving SMAD proteins.

suggesting that EVs secreted within the cornea may play a functional role during corneal homeostasis and wound healing [40]. In our studies, we have found that corneal wounding promotes EV release by the migrating epithelium in rat and rabbit injury models [41]. EVs released by the corneal epithelium postepithelial debridement appear to be restricted to the apical side of the basement membrane; however, in a keratectomy wound, EVs freely disperse into the stroma in the absence of a basement membrane (Fig. 3). These results suggest that the basement membrane may provide an important structural barrier that may prevent passage of EVs. We have also identified the presence of EVs in corneal stromal-endothelial co-cultures and the diffusion of EVs through the Descemet's membrane in rabbit corneas ex vivo [42]. In terms of bioactivity, epithelial-cell derived EVs isolated in vitro appear to promote increased fibroblast proliferation and angiogenesis, as assessed using aortic tissue explants [41]. While diffusion of pro-fibrotic factors, such as TGF- $\beta 1$ and TSP-1, may also be restricted by the basement membrane [16], diffusion of EVs by this laminin-rich barrier may be a key feature that determines whether the cornea develops a scar or regenerates postinjury. The corneal microenvironment following injury is permeated by the expression and secretion of a number of factors that promote wound repair. Further investigation of the interplay between free, unbound pro-fibrotic factors, and EVs during corneal wound healing is warranted to identify if these encapsulated vesicles mediate differential responses that may be key to myofibroblast persistence and scar development.

\section{Summary}

\section{TGF- $\beta 1$}

- Secreted as an inactive form bound to the latencyassociated protein (LAP).

\section{TSP-1}

- Secreted as a homotrimeric protein that may bind ECM proteins, including heparan sulfate proteoglycans, collagen, fibronectin, as well as pro-TGF- $\beta 1$ and integrins.

- Increased expression by the corneal epithelium following debridement or keratectomy.

- May promote myofibroblast differentiation by activating TGF- $\beta 1$ via binding to the Leu-Ser-Lys-Leu (LSKL)domain of LAP.

- In the absence of TSP-1, a reduced number of myofibroblasts are present in the corneal stroma following keratectomy or penetrating incision.

\section{av $\beta 6$}

- Integrin upregulated by the corneal epithelium following debridement, thereby aiding in epithelial-cell migration.

- Activates pro-TGF- $\beta 1$ by binding to the Arg-Gly-Asp (RGD)-domain of LAP.

- In the absence of $\alpha \nu \beta 6$, a delay in myofibroblast differentiation is observed following keratectomy or penetrating incision.

\section{EVs}

- Membrane-bound vesicles secreted by the corneal epithelium postdebridement or keratectomy.

- Migration appears restricted by the epithelial basement membrane.

- May promote fibroblast proliferation and angiogenesis. 
Funding The study is funded by NIH [R01EY005665 (JDZ), 5T32EY007145-20, and the NEI Core grant P30EY003790].

\section{Compliance with ethical standards}

Conflict of interest The authors declare that they have no conflict of interest.

Publisher's note Springer Nature remains neutral with regard to jurisdictional claims in published maps and institutional affiliations.

\section{References}

1. Pascolini D, Mariotti SP. Global estimates of visual impairment: 2010. Br J Ophthalmol. 2012;96:614-8.

2. Whitcher JP, Srinivasan M, Upadhyay MP. Corneal blindness: a global perspective. Bull World Health Organ. 2001;79:214-21.

3. Rittie L, Hutcheon AEK, Zieske JD. Mouse models of corneal scarring. Methods Mol Biol. 2017;1627:117-22.

4. Stepp MA, Zieske JD, Trinkaus-Randall V, Kyne BM, Pal-Ghosh $\mathrm{S}$, Tadvalkar G, et al. Wounding the cornea to learn how it heals. Exp Eye Res. 2014;121:178-93.

5. Pal-Ghosh S, Blanco T, Tadvalkar G, Pajoohesh-Ganji A, Parthasarathy A, Zieske JD, et al. MMP9 cleavage of the $\beta 4$ integrin ectodomain leads to recurrent epithelial erosions in mice. J Cell Sci. 2011;124:2666-75.

6. Blanco-Mezquita JT, Hutcheon AE, Stepp MA, Zieske JD. alphaVbeta6 integrin promotes corneal wound healing. Investig Ophthalmol Vis Sci. 2011;52:8505-13.

7. Torricelli AAM, Singh V, Santhiago MR, Wilson SE. The corneal epithelial basement membrane: structure, function, and disease. Investig Ophthalmol Vis Sci. 2013;54:6390-400.

8. Mohan RR, Hutcheon AEK, Choi R, Hong J, Lee J, Mohan RR, et al. Apoptosis, necrosis, proliferation, and myofibroblast generation in the stroma following LASIK and PRK. Exp Eye Res. 2003;76:71-87.

9. Jester JV, Petroll WM, Barry PA, Cavanagh HD. Expression of alpha-smooth muscle (alpha-SM) actin during corneal stromal wound healing. Investig Ophthalmol Vis Sci. 1995;36:809-19.

10. Connon CJ, Meek KM. The structure and swelling of corneal scar tissue in penetrating full-thickness wounds. Cornea. 2004; 23:165-71.

11. Tomasek JJ, Gabbiani G, Hinz B, Chaponnier C, Brown RA. Myofibroblasts and mechano-regulation of connective tissue remodelling. Nat Rev Mol Cell Biol. 2002;3:349-63.

12. Jester JV, Moller-Pedersen T, Huang J, Sax CM, Kays WT, Cavangh HD, et al. The cellular basis of corneal transparency: evidence for 'corneal crystallins'. J Cell Sci. 1999;112:613-22.

13. Zhang HY, Phan SH. Inhibition of myofibroblast apoptosis by transforming growth factor beta(1). Am J Respiratory Cell Mol Biol. 1999;21:658-65.

14. Yang X, Chen B, Liu T, Chen X. Reversal of myofibroblast differentiation: a review. Eur J Pharmacol. 2014;734:83-90.

15. Lassance L, Marino GK, Medeiros CS, Thangavadivel S, Wilson SE. Fibrocyte migration, differentiation and apoptosis during the corneal wound healing response to injury. Exp Eye Res. 2018;170:177-87.

16. Medeiros CS, Marino GK, Santhiago MR, Wilson SE. The corneal basement membranes and stromal fibrosis. Invest Ophthalmol Vis Sci. 2018;59:4044-53.

17. Dickson MC, Martin JS, Cousins FM, Kulkarni AB, Karlsson S, Akhurst RJ. Defective haematopoiesis and vasculogenesis in transforming growth factor-beta 1 knock out mice. Development. 1995;121:1845-54.

18. Sellheyer K, Bickenbach JR, Rothnagel JA, Bundman D, Longley MA, Krieg T, et al. Inhibition of skin development by overexpression of transforming growth factor beta 1 in the epidermis of transgenic mice. Proc Natl Acad Sci. USA. 1993; 90:5237-41.

19. Wyss-Coray T, Feng L, Masliah E, Ruppe MD, Lee HS, Toggas $\mathrm{SM}$, et al. Increased central nervous system production of extracellular matrix components and development of hydrocephalus in transgenic mice overexpressing transforming growth factor-beta 1 . Am J Pathol. 1995;147:53.

20. Vesaluoma M, Teppo A-M, Grönhagen-Riska C, Tervo T. Release of TGF- $\beta 1$ and VEGF in tears following photorefractive keratectomy. Curr Eye Res. 1997;16:19-25.

21. Zieske JD, Hutcheon AE, Guo X, Chung E-H, Joyce NC. TGF- $\beta$ receptor types I and II are differentially expressed during corneal epithelial wound repair. Investig Ophthalmol Vis Sci. 2001;42:1465-71.

22. Jester JV, Barry-Lane PA, Cavanagh HD, Petroll WM. Induction of alpha-smooth muscle actin expression and myofibroblast transformation in cultured corneal keratocytes. Cornea. 1996; 15:505-16.

23. Miyazono K. Positive and negative regulation of TGF-beta signaling. J Cell Sci. 2000;113:1101-9.

24. Brunet C, Sharpe P, Ferguson M. Inhibition of TGF-beta 3 (but not TGF-beta 1 or TGF-beta 2) activity prevents normal mouse embryonic palate fusion. Int J Developmental Biol. 2002; 39:345-55.

25. Shah M, Foreman DM, Ferguson M. Neutralisation of TGF-beta 1 and TGF-beta 2 or exogenous addition of TGF-beta 3 to cutaneous rat wounds reduces scarring. J Cell Sci. 1995;108:985-1002.

26. Gupta S, Rodier JT, Sharma A, Giuliano EA, Sinha PR, Hesemann NP, et al. Targeted AAV5-Smad7 gene therapy inhibits corneal scarring in vivo. PLOS One. 2017;12:e0172928.

27. Blanco-Mezquita JT, Hutcheon AE, Zieske JD. Role of thrombospondin-1 in repair of penetrating corneal wounds. Investig Ophthalmol Vis Sci. 2013;54:6262-8.

28. Wu W, Hutcheon AEK, Sriram S, Tran JA, Zieske JD. Initiation of fibrosis in the integrin Av $\beta 6$ knockout mice. Exp Eye Res. 2019;180:23-8.

29. Lawler J. The functions of thrombospondin-1 and-2. Curr Opin Cell Biol. 2000;12:634-40.

30. Crawford SE, Stellmach V, Murphy-Ullrich JE, Ribeiro SM, Lawler J, Hynes RO, et al. Thrombospondin-1 is a major activator of TGF- $\beta 1$ in vivo. Cell. 1998;93:1159-70.

31. Murphy-Ullrich JE, Poczatek M. Activation of latent TGF-beta by thrombospondin-1: mechanisms and physiology. Cytokine Growth Factor Rev. 2000;11:59-69.

32. Matsuba M, Hutcheon AE, Zieske JD. Localization of thrombospondin-1 and myofibroblasts during corneal wound repair. Exp Eye Res. 2011;93:534-40.

33. Hohenester E, Yurchenco PD. Laminins in basement membrane assembly. Cell Adh Migr. 2013;7:56-63.

34. Scheef EA, Huang Q, Wang S, Sorenson CM, Sheibani N. Isolation and characterization of corneal endothelial cells from wild type and thrombospondin-1 deficient mice. Mol Vis 2007; 13:1483-95.

35. Stepp MA. Corneal integrins and their functions. Exp Eye Res. 2006;83:3-15.

36. Fontana L, Chen Y, Prijatelj P, Sakai T, Fassler R, Sakai LY, et al. Fibronectin is required for integrin alphavbeta6-mediated activation of latent TGF-beta complexes containing LTBP-1. FASEB J. 2005;19:1798-808.

37. Munger JS, Huang X, Kawakatsu H, Griffiths MJD, Dalton SL, $\mathrm{Wu} \mathrm{J}$, et al. A mechanism for regulating pulmonary inflammation 
and fibrosis: the integrin $\alpha v \beta 6$ binds and activates latent TGF $\beta 1$. Cell.1999;96:319-28.

38. Raposo G, Stoorvogel W. Extracellular vesicles: exosomes, microvesicles, and friends. J Cell Biol. 2013;200:373-83.

39. Leszczynska A, Kulkarni M, Ljubimov AV, Saghizadeh M. Exosomes from normal and diabetic human corneolimbal keratocytes differentially regulate migration, proliferation and marker expression of limbal epithelial cells. Sci Rep. 2018;8:15173.

40. Han KY, Dugas-Ford J, Seiki M, Chang JH, Azar DT. Evidence for the involvement of MMP14 in MMP2 processing and recruitment in exosomes of corneal fibroblasts. Investig Ophthalmol Vis Sci. 2015;56:5323-9.
41. Han KY, Tran JA, Chang JH, Azar DT, Zieske JD. Potential role of corneal epithelial cell-derived exosomes in corneal wound healing and neovascularization. Sci Rep. 2017;7:40548.

42. Zieske JD, Hutcheon AEK, Guo X. Extracellular vesicles and cell-cell communication in the cornea. Anat Rec. 2019;1-8. [Epub ahead of print]

43. Annes JP, Munger JS, Rifkin DB. Making sense of latent TGF $\beta$ activation. J Cell Sci. 2003;116:217-24.

44. Munger JS, Huang X, Kawakatsu H, Griffiths MJ, Dalton SL, Wu $\mathrm{J}$, et al. The integrin alpha v beta 6 binds and activates latent TGF beta 1: a mechanism for regulating pulmonary inflammation and fibrosis. Cell.1999;96:319-28. 